Archivos de Ciencias de la Educación, Vol. 11, n 12, diciembre 2017, e034. ISSN 2346-8866 Universidad Nacional de La Plata.

Facultad de Humanidades y Ciencias de la Educación.

Departamento de Ciencias de la Educación.

\title{
Las incivilidades en la escuela. Potencialidades y recaudos del uso de la categoría en la investigación educativa
}

\author{
Incivilities at schools. Potentialities and precautions in the use of the \\ category for educational research
}

\section{Agustina Mutchinick}

Instituto de Investigaciones en Ciencias de la Educación- CONICET- Universidad de Buenos Aires, Argentina | agustinamutchinick@gmail.com

\section{PALABRAS CLAVE}

Incivilidades

Violencia

Escuela

Investigación educativa

KEYWORDS

Incivilities

Violence

School

Educational research

\section{RESUMEN}

La categoría incivilidades en la escuela es un concepto que diversos investigadores utilizan al abordar la temática de la violencia en el espacio escolar. Con el objetivo de correrse de miradas que solo contemplan la agresión física o los actos tipificados en el código penal, estos trabajos estudian las violencias cotidianas insertas en las interacciones sociales del sistema escolar (las transgresiones a las normas de convivencia, a las reglas del buen comportamiento, oposición entre estilos de socialización, etc.). Este artículo, a partir de una revisión crítica de las investigaciones que han utilizado la categoría, pretende dar cuenta de sus principales aportes, así como los recaudos que consideramos necesario tener en cuenta para su uso en la investigación de las prácticas educativas.

\section{ABSTRACT}

The category incivilities at schools is a concept which several researchers use when addressing the issue of violence at school place. In order to go beyond of the physical aggression or the acts typified in the penal code, these studies analyse the daily violence embedded in the social interactions of the school system (transgressions to norms of coexistence, rules of good behaviour, opposition to different syles of socialization, etc.). Based on a critical review of the researches that have used the category of incivilities, this article, attempts to show not only the main contributions of this category but also the need to be cautious of its use in research on educational practices. 


\section{Introducción}

Las violencias en la escuela es una temática que en los últimos años ha tenido una fuerte relevancia social y ha sido abordada desde diversos ámbitos: medios de comunicación $\underline{1}$, políticas públicas y de intervención ${ }^{2}$, espacio académico. Como objeto de estudio dentro del campo de la sociología en general y de la sociología de la educación en particular, su génesis en el plano internacional puede ubicarse en la década de los '70 y comienzos de los '80 en países como Estados Unidos y Francia. Sin embargo, es en la década de los '90 cuando comienza a consolidarse un abordaje más sistemático de la problemática, sumándose a estas dos naciones pioneras Inglaterra, España, México y Brasil, entre otros (Kaplan y García, 2006).

En la Argentina, con la excepción de algunos trabajos anteriores (Duschatzky, 1999; Kornblit, Mendes Diz y Frankel, 1991), hace poco más de una década que comenzaron a desarrollarse estudios sobre las violencias en la escuela, provenientes tanto de centros gubernamentales como de instituciones académicas, en especial, desde el ámbito de las universidades (Alterman y Foglino, 2005; Bleichmar, 2008; D' Angelo y Fernández, 2011; Di Leo, 2009, 2010, 2011a y 2011b; Kaplan, 2006 y 2009; Kaplan, Krotsch y Orce, 2012; Kornblit, 2008; Míguez, 2008; Míguez, Gallo y Tomasini, 2015; Noel, 2006 y 2009; Observatorio Argentino de Violencia en las Escuelas, 2008, 2010, 2013 y 2014; Paulín, 2015; Paulín, Tomasini y García Bastán, 2011; entre otros).

Una categoría a la que recurren diversos investigadores que abordan la temática de la violencia en la escuela es la noción de incivilidades. "Se trata de un concepto cuya utilización se ha prácticamente naturalizado en los países que más investigación desarrollan sobre esta problemática” (Piña, Furlán y Sañudo, 2003, p. 251). Como veremos, los trabajos han retomado esta noción en un intento por correrse de miradas restringidas de la violencia y contemplar determinados fenómenos que no se encuentran enmarcados en la agresión física o en los actos tipificados en el código penal.

El presente trabajo, a partir de una revisión crítica de las investigaciones que han utilizado la categoría, pretende problematizar su pertinencia para el análisis de las prácticas al interior de la vida escolar. Las preguntas que orientan el trabajo son: ¿Qué se entiende por incivilidades en la escuela? ¿Cuáles son las principales investigaciones sobre la temática? ¿Qué potencialidades ofrece esta conceptualización para el estudio de las violencias en el espacio escolar? ¿Qué recaudos son necesarios para su uso en la investigación de las prácticas educativas?

\section{Incivilidades en la escuela: los principales referentes en las investigaciones educativas}

La idea de incivilidad, como directriz para el análisis de la violencia escolar, tuvo gran repercusión, sobre todo en los estudios procedentes de Francia (Abramovay, 2005). Uno de los autores que ha abordado el concepto y lo ha popularizado en el campo de la investigación educativa es Eric Debarbieux. Su definición de la violencia en el medio escolar presenta tres dimensiones (Debarbieux, 1996):

1- los crímenes y delitos: robos, golpes y heridas, tráfico y uso de drogas, etc. Aquello que se encuentra tipificado en el Código Penal;

2- las incivilidades: refieren a situaciones que trasgreden los códigos elementales de la vida en sociedad, el código de las "buenas costumbres". No son necesariamente comportamientos ilegales, en el sentido jurídico, sino infracciones al orden concebido en los encuentros de la vida cotidiana. Son violencias cotidianas, repetidas (Debarbieux, 2008; Debarbieux, Garnier, Montoya y Tichit, 1999). Ellas pueden ser del orden del ruido, de la suciedad, de la ofensa, de todo lo que cause desorden.

3- el sentimiento de inseguridad: aquello que llama el "sentimiento de violencia”, que resulta de 
los dos componentes precedentes, pero proviene igualmente de un sentimiento más general relacionado con los diversos contextos sociales de referencia.

No todas las dimensiones tienen la misma preponderancia: la violencia en el espacio escolar no se refiere tanto al mundo del delito como al de las incivilidades. La escuela, sostiene este autor, se encuentra a resguardo de las "violencias brutales" siendo "lo impalpable, el ambiente, las incivilidades, lo que construye la inseguridad y genera un sentimiento de impunidad" (Debarbieux, 1996, p. 40). Las incivilidades pueden parecer detalles anodinos, pero son demasiado repetidos como para no adquirir cierta significación. La mayoría de las veces la violencia es una cuestión de actos tenues y difícilmente perceptibles, pero frecuentemente repetidos.

Una cuestión central que subraya este autor es que la incivilidad que se revela en la escuela, no debe ser pensada como un conflicto entre "bárbaros” y "civilizados": la incivilidad no es la no-civilización, ni la “mala educación”. Más que el enfrentamiento entre "barbarie y civilización” o "naturaleza y cultura”, se trata de "conflicto de civilidades” (Debarbieux, 2001), de una oposición entre estilos de socialización que derivan de las diferencias generacionales, sociales, culturales (Debarbieux et al, 1999). Asimismo, Charlot y Emin (1997) hacen referencia a que profesores y alumnos presentan códigos culturales y maneras de comportarse diferentes, lo cual es percibido por los actores como violencias cotidianas.

Otros autores que dan cuenta del predominio de las incivilidades en el mundo escolar son Cécile Carra y François Sicot (1997). En su estudio sobre las victimizaciones de estudiantes de colegios del departamento de Doubs (Francia), afirman que las formas de violencia en la escuela se reparten entre dos grandes polos: los atentados a la integridad física y las incivilidades y que son estas últimas las que presentan mayor frecuencia $a^{3}$.

Bernard Charlot (2002) también aborda el concepto de incivilidades. En sus trabajos hace referencia a una distinción que realizan algunos sociólogos franceses que investigan la violencia en el espacio escolar: la diferenciación entre violencia, trasgresión e incivilidad. El término violencia remite a las violaciones de la ley con utilización o amenaza de utilización de la fuerza física (lesiones, extorsiones, tráfico de droga, insultos graves). Las transgresiones refieren a los comportamientos contrarios a las regulaciones internas del establecimiento (no es ilegal desde el punto de vista de la ley: absentismo, no realización de trabajos escolares, faltas de respeto, etc.). Por su parte, las incivilidades no contradicen ni la ley ni el reglamento interno del establecimiento, sino las reglas de la buena convivencia (desorden, groserías, palabras ofensivas, generalmente ataques cotidianos -y con frecuencia repetidos- al derecho de cada uno de ser respetado). Al igual que sus compatriotas, refiere a la preponderancia de las incivilidades frente a los golpes, el uso de armas, los abusos sexuales (Charlot, 1997).

Trabajos sobre la violencia en las escuelas de otros países del viejo continente también han utilizado el concepto incivilidades. Podemos mencionar el estudio de Alain Clémence (2002) en Suiza que las define como "actos que perturban el buen funcionamiento de la escuela a partir del quiebre de las reglas del buen comportamientos” (p. 229) 4 y los trabajos de Janine Blomart (2002) en Bélgica y Nunes, Caridade y Sani (2015) en Portugal que las entienden como "acciones antisociales” cotidianas.

Por su parte, en América Latina este término ha tenido una importante acogida. En México las investigaciones de Saucedo Ramos y Mejía Hernández (Mejía Hernández, 2012; Saucedo Ramos y Mejía Hernández, 2013) hacen referencia a las incivilidades entendiéndolas como "la puesta en marcha (o el intercambio) de pequeñas violencias cotidianas que atacan el derecho que cada uno tiene de ser respetado" (Saucedo Ramos y Mejía Hernández, 2013, p. 174). También encontramos desarrollada esta categoría, a partir de las definiciones de los estudios franceses, en el estado del arte que realizan Piña, Furlán y Sañudo $(2003)^{5}$. 
En Brasil, Tavares dos Santos (2001 y 2002) en su estudio sobre la violencia en el espacio escolar en la ciudad de Porto Alegre refiere al concepto de incivilidades como "conflicto de civilidades" y sostiene que "entre profesores y alumnos existen portadores de diferentes normas de conducta, lo cual se manifiesta en formas menores, moleculares y cotidianas de violencia en el espacio escolar” (p. 109) ${ }^{6}$. Con ello el autor hace referencia a las dificultades que se establecen en la convivencia entre grupos sociales que utilizan diversos códigos culturales en sus relaciones sociales. Por su parte, Abramovay definen la categoría como aquellos actos que "contradicen las reglas de la buena convivencia” (Abramovay, Cunha y Pinto Calaf, 2009, p. 23), diferenciándolas de las "violencias duras" que están presentes en los códigos penales y las "violencias simbólicas”. Sostiene que las incivilidades son cada vez más comunes (Abramovay, 2006) y más frecuentes que la "violencia dura" (Abramovay y Rua, 2002). También desde Uruguay, encontramos trabajos que abordan esta dimensión de las violencias, entre los que destacamos especialmente los de Nilia Viscardi (Viscardi, 2003; Viscardi y Alonso, 2013).

En la Argentina, los relevamientos llevados a cabo por el Observatorio Argentino de Violencia en las Escuelas en los años 2005 y 2007 hacen referencia al término incivilidades diferenciándolo de lo que llaman "las situaciones de violencia en sentido estricto"프. Entre las primeras se incluyen las burlas, los insultos, los gritos, las exclusiones y la rotura de útiles u otras pertenencias; mientras que las segundas contienen las amenazas de daño de un compañero, golpes o lastimaduras, amenazas o lesiones de patotas y robo por la fuerza o con amenazas.

Ana Lía Kornblit y su equipo en un estudio sobre la convivencia y los vínculos violentos entre adolescentes escolarizados de todo el país, distinguen entre:

1. el "hostigamiento" que incluye, por un lado, las transgresiones -comportamientos que van en contra de las reglas internas de la institución escolar (ausentismo, no realización de las tareas por parte de los alumnos, etc.)- y, por el otro, las incivilidades -las infracciones de las reglas de convivencia y los ataques cotidianos al derecho a ser respetado- $\mathrm{y}$

2. la "violencia en sentido estricto o extrema", que remite al uso de la fuerza para ejercer el poder o la dominación sobre el otro (lesiones, robos, extorsiones) (Kornblit, Adaszko, Mendes Diz, Di Leo y Camarotti, 2008)

El equipo dirigido por Carina Kaplan trabaja a partir de una conceptualización amplia de las violencias tomando en cuenta episodios de violencia graves $^{\underline{8}}$ e incivilidades $\underline{9}$ de manera de integrar así "situaciones contempladas en los marcos legales y situaciones que implican básicamente fricciones sobre las convenciones que regulan los usos y comportamientos en los espacios públicos” (García, 2010, p. 75).

Cabe mencionar que las investigaciones argentinas relevadas coinciden con las europeas y con aquellas de otros países latinoamericanos al sostener el predominio de las situaciones catalogadas como incivilidades frente a otros hechos de violencia en sentido estricto.

La revisión de estos estudios nos permite dar cuenta de los trabajos empíricos más relevantes que se han realizado en los últimos años sobre las incivilidades en el ámbito escolar, así como las definiciones y conceptualizaciones que ha adquirido el término. A continuación, intentaremos dar cuenta de los principales aportes de la categoría, así como los recaudos que consideramos que es necesario tener en cuenta si se pretende abordarla empíricamente. 


\section{Contribuciones y recaudos}

Uno de los "beneficios” de la categoría es que permite establecer distinciones analíticas en el marco de una temática tan amplia como la de las violencias en la escuela, que abarca "conductas extremadamente heterogéneas” (Dubet, 2003) que responden a lógicas diversas (insultos y burlas, amenazas, golpes, uso de armas, destrucción y robo de bienes materiales, y otras tantas prácticas que los actores escolares definen como violentas). Como señala Kaplan (2006) "La falta de distinción sistemática entre los tipos de violencia práctica hace que no se puedan interpretar sus efectos sociales diferenciales, lo cual consideramos, resulta un obstáculo epistemológico que los propios investigadores debemos afrontar” (p. 21).

Es una idea extendida entre los autores que trabajan esta problemática la necesidad de dar cuenta de la diversidad de fenómenos y expresiones que abarca el término "violencia en la escuela” o "violencia escolar" (Charlot, 2002; Debarbieux, 1996; Dubet, 2003; Kornblit, 2008; Míguez y Tisnes, 2008; Parra Sandoval, 1992; Observatorio Argentino de Violencia en las Escuelas, 2014). La categoría incivilidades es una de las distinciones propuestas en este sentido. Ella nos permite corrernos de perspectivas que equiparan la violencia en las instituciones educativas con la violencia física o la delincuencia y poner el foco en prácticas escolares y modos de sociabilidad como los insultos, burlas, apodos ofensivos, discriminaciones, humillaciones, entre otras, que tienen una especificidad propia, que no amenazan de manera directa la integridad física de los actores de la vida institucional, pero exhiben una gran ubicuidad en el espacio escolar y tienen efectos simbólicos en la construcción de la experiencia escolar y en la autoestima social y educativa de los sujetos que las sufren. Como sostienen Viscardi y Alonso (2013):

... ha sido el establecimiento del concepto de incivilidades el que confirió su especificad a un objeto que, de otro modo, podría subsumirse por entero a los hechos analizados generalmente en términos criminológicos o psicológicos, permitiendo afinar y ampliar la descripción de los fenómenos de violencia y conflicto en el espacio escolar (Viscardi \& Alonso, 2013, p. 91).

Si bien en los últimos años se ha instalado en la opinión pública la percepción de que la escuela, y especialmente la escuela secundaria de gestión estatal, se encuentra atravesada por la violencia física, como pudimos observar en las investigaciones precedentes, son prácticas denominadas como incivilidades las que dan cuenta de manera más pertinente de las violencias cotidianas insertas en las interacciones sociales del sistema escolar (Abramovay y Rua, 2002; Blomart, 2002; Carra y Sicot, 1997; Charlot, 1997; Debarbieux, 1996, 2001, 2002; Debarbieux et al, 1999; García, 2010; Kaplan 2009; Kornblit, Adaszko, Mendes Diz, Di Leo y Camarotti, 2008; Observatorio Argentino de Violencia en las Escuelas, 2008 y 2010; Viscardi, 2003; Viscardi y Alonso, 2013).

Ahora bien, esta noción presenta algunas cuestiones que consideramos que deben ser tenidas en cuenta si se pretende abordarla empíricamente. Por un lado, consideramos que las definiciones propuestas requieren mayores precisiones ya que por momentos los márgenes entre lo que se considera o no incivilidades son inaprensibles. La definición a partir de su contraposición con las prácticas ilegales es un ejemplo de estas ambigüedades ya que encontramos situaciones y acciones, como los insultos, que son consideradas por gran parte de los investigadores como incivilidades al tiempo que están catalogadas en el código penal $\underline{10}$. Sin pretender confinar la categoría a los límites de una definición estricta, consideramos que ella presenta algunas imprecisiones que dificultan su abordaje empírico. Por otro lado, entendemos que es necesario tener en cuenta la procedencia del concepto ya que como veremos, este tiene origen en el campo de la criminología; lo cual puede generar un sesgo criminalizante sobre las prácticas escolares.

El término incivilidad que autores como Debarbieux o Abramovay adoptan para el ámbito escolar es retomado de los trabajos sobre la seguridad pública del politólogo francés Sebastián Roché. Para este último, las incivilidades desafían los códigos elementales de la vida colectiva. "Se trata de ataques al orden público" 
(Roché, 1994, s/p), los cuales son percibidos como una molestia, pero no necesariamente son calificados como delitos. Son incivilidades los gestos obscenos, los insultos, las amenazas, las paredes manchadas por los graffittis, el vandalismo, las ventanas rotas, los ciclomotores o coches quemados, el consumo de drogas y alcohol en espacios públicos, la contaminación sonora, entre otras (Roché, 1994 y 1996). Ellas se viven como una falta de respeto y un ataque a los derechos elementales y "constituyen los marcadores del desorden que impulsan al individuo a ponerse en alerta”11 (Roché, 1994, s/p).

Según el enfoque de Roché, las incivilidades se vinculan tanto con el sentimiento de inseguridad como con el delito ya que pueden generar un aumento del miedo a la vez que ser el punto de partida de la delincuencia en la calle (Roché, 1996, 2002). En este sentido, el autor sostiene que las causas de la delincuencia no se podrían deducir de macrofactores como la pobreza o la inestabilidad habitacional sin tener en cuenta las incivilidades (Roché, 1994) $\underline{12}$. Estas impulsan a los sujetos fuera de la vida social y al repliegue sobre sí y favorecen la desconfianza hacia instituciones tales como la policía, la justicia, etc., lo cual colabora en la conformación de un espacio social más proclive al delito (Roché, 1996).

Es crucial señalar que es fundamentalmente sobre las "conductas incivilizadas" donde se asienta la famosa política de tolerancia cero; la cual pretende "detectar los casos de predelincuencia y tratarlos antes de que se transformen en delitos” (Alaín Pérez, 1999 citado en Pegoraro, 2002, p. 35) Son este tipo de políticas las que entienden que los problemas de seguridad deben resolverse con mayor dureza y represión policial, aun cuando ello implique el sacrificio de derechos y garantías $\underline{13}$ (Pegoraro, 2002).

Lo que nos interesa poner en evidencia es que se incorpora al campo educativo una categoría fuertemente ligada a políticas punitivas de la seguridad pública. Esta extrapolación puede generar un sesgo criminalizante sobre las prácticas escolares y el riesgo de abordarlas desde una mirada judicial que lejos de intentar comprender las circunstancias que las originan, tenga como propósito castigar las conductas que se corren de la norma $\frac{14}{}$. Como afirman Debarbieux et al (1999) si se confunde "toda puesta en duda del orden con una suerte de predelincuencia (p. 63) el uso del concepto de incivilidad puede servir para justificar la represión.

Por último, señalemos que algunos autores se han distanciado del concepto de incivilidades por considerar que entraña un sesgo etnocéntrico (Observatorio Argentino de Violencia en las Escuelas, 2013) o que puede dar lugar a interpretaciones de naturaleza evolucionista y ser utilizado para estigmatizar a individuos y a poblaciones que no se comportan según las reglas hegemónicas (Debarbieux, 2002, 2008). De esta manera, puede apreciarse en los últimos años, investigaciones que, intentando dar cuenta de prácticas y procesos enmarcados dentro de la categoría incivilidades, utilizan otros términos como los de microviolencias o “malos tratos”. Al respecto, García Bastán (2016) afirma que "no existe una distancia intransitable" entre la lectura de los conflictos en la escuela como "conflicto entre civilidades" y ciertas percepciones "nativas" de corte culturalista que responsabilizan a las familias de los jóvenes por una socialización percibida como deficitaria. Sostiene que:

Afirmar a modo de declaración de principios que referir a incivilidades no implica pensar en una lucha de "bárbaros" contra "civilizados" es necesario pero puede resultar insuficiente si en nuestros análisis emprendemos luego contra cualquier violencia menor (o micro-violencia si se prefiere) apelando a presuntas distancias socio-culturales entre la escuela y las poblaciones que asisten a ella (García Bastán, 2016, p. 11).

Desde nuestra perspectiva, no coincidimos con esta argumentación ya que consideramos que la referencia a la idea de "conflicto de civilidades" no apunta a establecer una responsabilización de los sectores populares, sino que intenta llamar la atención sobre el desencuentro que experimentan los actores escolares por sus diversas y en ocasiones, antagónicas maneras de estar y comportarse $\mathrm{in}^{15}$. 
Disentimos con el cariz evolucionista y etnocéntrico que los autores intentan asociar a la categoría ya que consideramos que la caracterización de las prácticas y modos de sociabilidad como civil o incivil varía según el contexto espacio temporal, la trama institucional y las historias y posiciones sociales de los sujetos. Las incivilidades siempre se refieren a un parámetro de normalización que se modifica en el largo plazo y difiere en cada configuración particular (Elias, 1987). No hay un patrón universal sobre qué prácticas son inciviles. Las violencias y las incivilidades presentan en cada época y cultura un sentido propio de manera que la sensibilidad respecto a lo que está y no está permitido se transforma y muta en los diferentes contextos. De esta manera, lejos de reforzar un discurso de naturaleza evolucionista, consideramos que los comportamientos deben ser comprendidos en el marco de un contexto social e institucional y en marco de biografías particulares.

\section{Conclusiones}

La categoría incivilidades es abordada por una importante cantidad de investigaciones tanto de Europa como de América Latina, las cuales la definen centralmente como transgresiones a las normas de convivencia, a las reglas del buen comportamiento, oposición entre estilos de socialización y códigos culturales, acciones antisociales, pequeñas violencias cotidianas, ataques al derecho a ser respetado. En ocasiones es delimitada por la negativa, contrapuestas a la violencia física o a prácticas delictivas enmarcadas en el código penal. Lo que se observa es que la mayoría de los estudios recuperan las conceptualizaciones que realizan Debarbieux y Charlot (Abramovay et al., 2009; Clémence, 2002; Kornblit, 2008; Nunes et al., 2015; Saucedo Ramos y Mejía Hernández, 2013; Tavares dos Santos, 2002; Viscardi y Alonso, 2013) por lo cual se entiende que no existan marcadas diferencias entre los autores.

El artículo pretendió dar cuenta de las potencialidades del concepto y algunos recaudos para su uso. Entre las primeras encontramos que permite establecer distinciones analíticas y céntranos en dimensiones de la violencia en la escuela que podrían quedar veladas frente a situaciones como el uso de armas, los robos, el abuso sexual, etc. Esta noción procura visibilizar las prácticas cotidianas por sobre las excepcionales; lo cual se torna central, sobre todo si tenemos en cuenta que a pesar de su periodicidad (o a causa de ella), dichas prácticas suelen en ocasiones pasar inadvertida por las autoridades escolares (Abramovay et al., 2009 y D' Angelo y Fernández, 2011, Autor, 2013). Los recaudos mencionados se refieren, por un lado, a tener en cuenta que algunas de las conceptualizaciones de la categoría dificultan establecer qué prácticas y situaciones pueden enmarcarse en ella. Nuestros trabajos han avanzado en una caracterización de las incivilidades en la escuela que consideramos nos permite abordarlas empíricamente. Las entendemos como prácticas y modos de sociabilidad ubicuos en el espacio escolar que no representan una amenaza a la integridad física pero que son vividos como ofensas por los individuos y grupos que las padecen. Por último, queremos subrayar que la procedencia del concepto del campo del delito no inhabilita su utilización en el estudio de las prácticas educativas, pero sí nos obliga a estar atentos para no analizarlas y abordarlas a partir de supuestos propios de los paradigmas hegemónicos de matriz criminalizante.

\section{Notas}

1 En los medios de comunicación, a partir de la espectacularización de ciertos episodios que excepcionalmente tienen lugar en la escuela (uso de armas, abusos sexuales, golpizas, vandalismo, etc.) se forja la creencia de que estos sucesos constituyen prácticas y vivencias habituales del espacio escolar. La escuela se presenta de esta manera como un lugar inseguro atravesado cotidianamente por la violencia física (Brener, 2009; Saez, 2015).

$\underline{2}$ En la órbita de las políticas gubernamentales, en nuestro continente así como en Europa se han 
desarrollado una gran cantidad de iniciativas para el abordaje de las violencias en la escuela (Krauskopf, 2006) A nivel nacional, cabe resaltar la creación en el año 2004 del Observatorio Argentino de Violencia en las Escuelas, y en el año 2013 la sanción de la Ley para la promoción de la convivencia y el abordaje de la conflictividad social en las instituciones educativa ( $\left.\mathrm{N}^{\circ} 26.892\right)$.

$\underline{3}$ Los principales hechos de los cuales los alumnos dicen ser víctimas son faltas de respeto (47,8 \%) y ruptura de relaciones personales $(27,7 \%)$.

4 Traducción propia

$\underline{5}$ Aunque se explicita allí claramente que hasta el año 2003 nadie utiliza la categoría en México.

$\underline{6}$ Traducción propia.

Z Los relevamientos se realizaron a través de una encuesta integrada al Operativo Nacional de Evaluación (ONE) que implementa la Dirección Nacional de Información y Estadística Educativa (DINIEE). El del año 2005 corresponde al informe del Observatorio Argentino de Violencia en las Escuelas de 2008 y el del año 2007 al informe del Observatorio Argentino de Violencia en las Escuelas de 2010. Como veremos más adelante, en el relevamiento realizado en 2010 se dejó de utilizar el término incivilidades por el de "malos tratos”.

$\underline{8}$ Portación de armas, agresiones físicas, robos, amenazas, etc.

$\underline{9}$ Las situaciones contempladas como incivilidades consistieron en: escritura de bancos y paredes, insultos entre alumnos, fumar en la escuela, faltas de respeto entre compañeros, desordenes generales que impiden el desarrollo de la clase, malas condiciones del colegio, no respetar símbolos patrios, humillaciones entre alumnos, ensuciar la escuela intencionalmente, daños de láminas y/o decoraciones de la escuela, faltas de respeto hacia los docentes o directivos, humillaciones de los estudiantes hacia las autoridades y escupirse entre compañeros.

10 Los insultos pueden ser vistos como delitos contra el honor. En el artículo 110 el código penal establece: "El que intencionalmente deshonrare o desacreditare a una persona física determinada será reprimido con multa de pesos mil quinientos (\$1.500.-) a pesos veinte mil (\$20.000.-). En ningún caso configurarán delito de injurias las expresiones referidas a asuntos de interés público o las que no sean asertivas. Tampoco configurarán delito de injurias los calificativos lesivos del honor cuando guardasen relación con un asunto de interés público".

11 Traducción propia.

12 Debarbieux no concuerda en este sentido con Roché y critica la negación de este autor de ligar la incivilidad a la crisis económica y al desempleo y su subestimación de la importancia de las desigualdades sociales ya que sostiene que la incivilidad y la inseguridad se encuentran determinadas socialmente (Debarbieux et al, 1999).

13 Pegoraro (2003) sostiene que esta política de tolerancia cero en los hechos solo persigue incivilidades dejando impune los grandes delitos del poder (sobre todo los económicos). Según el autor, se trataría de una política punitiva selectiva o de una política que, en términos de Foucault, administra diferencialmente los ilegalismos.

14 Estas teorías no son lejanas a la escuela. Las políticas de tolerancia cero también han calado hondo en el sistema educativo de varios países, entre los cuales algunos estados de Estados Unidos hacen bandera.

15 También discutimos con la idea de "conflicto de civilidades" pero los motivos son otros. Por nuestra parte, consideramos que el término conlleva el riesgo de homologar conflicto y violencia y por lo tanto, 
confundir todo desacuerdo entre códigos de conducta y estilos de socialización con prácticas violentas. Por el contrario, estimamos que el conflicto es constitutivo de la dinámica escolar -como de toda relación social- y si bien puede devenir en violencia, no tiene necesariamente relación directa con ella. La violencia y el conflicto no son términos que puedan equiparse (Simmel, 2013; Wieviorka, 2002).

\section{Bibliografía}

Abramovay, M. (2005). Violencia en las escuelas: un gran desafío. Revista Iberoamericana de Educación, (38). Recuperado de: http://www.rieoei.org/rie38a03.pdf

Abramovay, M. (Coord.). (2006). Cotidiano das escolas: entre violências. Brasília: UNESCO.

Abramovay, M., Cunha, A. L., e Pinto Calaf, P. (2009). Revelando tramas, descobrindo segredos: violência e convivência nas escolas. Brasilia: RITLA. Recuperado de: http://www.abglt.org.br/docs/Revelando Tramas.pdf

Abramovay, M., e Rua, M. das G. (2002). Violências nas escolas. Brasília: UNESCO.

Alterman, N. y Foglino, A. M. (2005). Los Centros de Actividades Juveniles: Balance de su operación como estrategia para mejorar la convivencia en las secundarias cordobesas. Revista Mexicana de Investigación Educativa, 10 (26), 669-692.

Bleichmar, S. (2008). Violencia Social-Violencia Escolar: De la puesta de límites a la construcción de legalidades. Buenos Aires: Novedades Educativas.

Blomart, J. (2002). Evitando a violência no ambiente das escolas primárias. En E. Debarbieuex e C. Blaya (Orgs.), Violência nas Escolas: dez abordagens européias (pp. 223-245). Brasília: UNESCO.

Brener, G. (2009). Violencia y escuela como espectáculo. La relación medios-sociedad. En C. Kaplan (Dir.), Violencia escolar bajo sospecha (pp. 199-140). Buenos Aires: Miño y Dávila.

Carra, C., et Sicot, F. (1997). Una autre perspective sur les violences scolaires: L'expérience de victimation. En J.C. Emin et B. Charlot (Eds.), Violences a l'ecole - etat des savoirs. (pp. 61-82). Paris: Armand Colin Mason.

Charlot, B. (1997). Violences scolaires: Représentations, pratiques et normes. En B. Charlot et J. C. Emim (Coords.), Violences a l'école: État des savoirs. París: Armand Colin.

Charlot, B. (2002). A violência na escola: Como os sociólogos franceses abordam essa questao. Sociologias, 8 (4), $432-443$.

Charlot, B. et Emin, J. C. (Coords.). (1997). Violences à l'école: État des savoirs. Paris: Armand Colin.

Clémence, A. (2002). Violência e incivilidade na escola: a situação na Suíça. En É. Debarbieuex, et C. Blaya (Orgs.), Violência nas Escolas: dez abordagens européias (pp. 223-245). Brasília: UNESCO.

D'Angelo, L. y Fernández, D. (2011). Clima, conflictos y violencia en la escuela. Buenos Aires: UNICEFFLACSO.

Debarbieux, E. (1996) La violence en milieu scolaire: État des lieux (Vol. 1). Paris: ESF éditeur.

Debarbieux, E. (2001). A violência na escola francesa: 30 anos de construção social do objeto (1967-1997). Educação e Pesquisa, 27 (1), 163-193.

Debarbieux, E. (2002). Cientistas, políticos e violência: rumo a uma comunidade científica européia para lidar com a violência nas escolas? En E. Debarbieux y C. Blaya (Orgs.), Violência nas escolas: Dez 
abordagens européias. (pp. 13-33). Brasília: Unesco.

Debarbieux, E. (2008). Les Dix commandements contre la violencia a l'ecole. París: Odile Jacob.

Debarbieux, E., Garnier, A., Montoya, Y. et Tichit, L. (1999). La violence en milieu scolaire: Le désordre des choses. (Vol. 2). París: ESF éditeur.

Di Leo, P. F. (2009). Subjetivación, violencias y climas sociales escolares: Un análisis de sus vinculaciones con experiencias de promoción de la salud en escuelas medias públicas de la Ciudad Autónoma de Buenos Aires (Tesis Doctoral inédita), Universidad de Buenos Aires, Argentina.

Di Leo, P. F. (2010). Discursos en torno a las violencias y la autoridad en escuelas medias públicas: Entre la desubjetivación y la integración normativa. Educación, Lenguaje y Sociedad, 7 (7), 53-78.

Di Leo, P. F. (2011a). Violencias y climas sociales en escuelas medias: Experiencias de docentes y directivos. Educação e Pesquisa, 37 (3), 599-612.

Di Leo, P. F. (2011b). Escuela y violencia: Desplegando una relación problemática. Revista de la Escuela de Ciencias de la Educación, (6), 231-249.

Dubet, F. (2003). Las figuras de la violencia en la escuela. Reflexiones Pedagógicas, (19) 27-37.

Duschatzky, S. (1999). La escuela como frontera: reflexiones sobre la experiencia escolar de los jóvenes de sectores populares. Buenos Aires: Paidós.

Elias, N. (1987). El proceso de la civilización: Investigaciones sociogenéticas y psicogenéticas. Madrid: Fondo de Cultura Económica.

García Bastán, G. (2016). Investigar cualitativamente las incivilidades en la escuela media. Reflexiones metodológicas desde perspectivas interaccionistas. Revista Latinoamericana de Metodología de las Ciencias Sociales, 6(1), 1-16. Recuperado de: http://www.relmecs.fahce.unlp.edu.ar/article/view/relmecs_v06n01a05

García, S. (2010.) Violencias, incivilidades y miedos en el ámbito educativo: Un estudio socioeducativo sobre la perspectiva de los estudiantes de educación secundaria (Tesis de Maestría inédita) Maestría en Educación con orientación en Gestión Educativa. Universidad de San Andrés, Escuela de Educación, Buenos Aires, Argentina.

Kaplan, C. (dir.). (2006). Violencias en plural: Sociología de las violencias en la escuela. Buenos Aires: Miño y Dávila.

Kaplan, C. (dir.). (2009). Violencia escolar bajo sospecha. Buenos Aires: Miño y Dávila.

Kaplan, C. y García, S. (2006). Las violencias en la escuela en el mundo: Un mapa de los estudios socioeducativos. En C. Kaplan (Dir.), Violencias en plural: Sociología de las violencias en la escuela. (pp. 105-204). Buenos Aires: Miño y Dávila.

Kaplan, C., Krotsch, L., y Orce, V. (2012). Con ojos de joven: Relaciones entre desigualdad, violencia y condición estudiantil. Buenos Aires: Editorial de la Facultad de Filosofía y Letras, Universidad de Buenos Aires.

Kornblit, A. L. (2008) Violencia escolar y climas sociales. Buenos Aires: Biblos.

Kornblit, A. L., Adaszko, D. Mendes Diz, A. M., Di Leo, P. F., y Camarotti, A. C. (2008). En A. L. Kornblit (Coord.). Violencia escolar y climas sociales. (pp. 43-58) Buenos Aires: Biblos.

Kornblit, A.L., Mendes Diz, A.M y Frankel, D. (1991). Manifestaciones de violencia en la escuela media. En F. Lolas (Comp.), Agresividad y violencia. Buenos Aires: Losada. 
Krauskopf, D. (2006). Estado del arte de los programas de prevención de la violencia en ámbitos escolares. Washington, D. C.: OPS.

Míguez, D. (comp.) (2008). Violencias y conflictos en las escuelas: aproximaciones a una problemática actual. Buenos Aires: Paidós.

Míguez, D., Gallo, P. y Tomasini, M. (Coord.). (2015). Las dinámicas de la conflictividad escolar. Procesos y casos en la Argentina reciente. Miño y Davila: Buenos Aires.

Míguez, D. y Tisnes, A. (2008). Midiendo la violencia en las escuelas argentinas. En D. Míguez (Ed.), Violencias y conflictos en las escuelas (pp. 33-71). Buenos Aires: Paidós.

Noel, G. (2006). Una aproximación etnográfica a la cotidianeidad, el conflicto y la violencia en escuelas de barrios populares. En Observatorio Argentino de Violencia en las Escuelas. Miradas interdisciplinarias sobre la violencia en las escuelas. Buenos Aires: Ministerio de Educación, Ciencia y Tecnología de la Nación, pp. 39-45

Noel, G., Gallo, P., y Miguez, D. (2009). Violencia en las escuelas desde una perspectiva cualitativa. Buenos Aires: Observatorio Argentino de Violencia en las Escuelas. Ministerio de Educación de la Nación.

Nunes, L. M., Caridade, S. e Sani, A. I. (2015). Avaliação do meio escolar: um estudo exploratório. Revista Lusófona de Educação, 30(30). 141-158.

Observatorio Argentino de Violencia en las Escuelas. (2008). Violencia en las escuelas: un relevamiento desde la mirada de los alumnos. Buenos Aires: Ministerio de Educación de la Nación.

Observatorio Argentino de Violencia en las Escuelas. (2010). Violencia en las escuelas: un relevamiento desde la mirada de los alumnos II. Buenos Aires: Ministerio de Educación de la Nación.

Observatorio Argentino de Violencia en las Escuelas. (2013). Relevamiento cuantitativo sobre violencia en las escuelas desde la mirada de los alumnos. Buenos Aires: Ministerio de Educación de la Nación

Observatorio Argentino de Violencia en las Escuelas (2014); Relevamiento estadístico sobre clima escolar, violencia y conflicto en escuelas secundarias según la perspectiva de los alumnos. Buenos Aires: Ministerio de Educación de la Nación.

Parra, R., González, A., Moritz, O., Blandón, A. y Bustamante, R. (1994). La escuela violenta. Colombia: Fundación Fes.

Paulín, H. (2015). "Ganarse el respeto": un análisis de los conflictos de la sociabilidad juvenil en la escuela secundaria. Revista mexicana de investigación educativa, 20(67), 1105-1130.

Paulín, H., Tomasini, M. y García Bastán, G. (junio, 2011). Luchas por el reconocimiento en la sociabilidad juvenil: Un análisis a partir de las peleas en escuelas secundarias. Trabajo presentado en VII Jornadas de Investigación en Educación: Encrucijadas de la educación: saberes, diversidad y desigualdad. Universidad Nacional de Córdoba, Córdoba.

Pegoraro, J. S. (2002). Las políticas de seguridad y la participación comunitaria en el marco de la violencia social. En R. Briceño León (Ed.). Violencia, sociedad y justicia en América Latina. (pp. 29-53) Buenos Aires: CLACSO.

Pegoraro, J. S. (2003). Una reflexión sobre la inseguridad. Argumentos. Revista de crítica social, (2), 1-7. Recuperado de: https://dialnet.unirioja.es/descarga/articulo/3991753.pdf

Piña, J. M., Furlán, A. y Sañudo, L. (2003) Acciones, actores y prácticas educativas, v.2. México: Grupo Ideograma. 
Roché, S. (1994). Les incivilités, défis à l'ordre social : Insécurités urbaines. Projet. (238) 37-46.

Roché, S. (1996). Les incivilités vues du côté des institutions: modes d'action, traitements et circuits.

Roché, S. (2002). Tolérance zéro? Incivilités et insécurité. Paris: Odile Jacob.

Saez, V. (2015). Una mirada a la investigación sobre medios, violencia y escuela. Entramado, 11(1), 136155. http://doi.org/10.18041/entramado.2015v11n1.21117

Saucedo Ramos, C. y Mejía Hernández, J. M. (2013). ¿Es posible la convivencia escolar? Aportes analíticos desde la realidad de la escuela en nuestros días. En X. Martínez y D. Rosado (Coords.), Estudios de la juventud y filosofía de la no violencia: conciencia generacional, ciudadanía y argumentación. México D.F.: Paideia Siglo XXI.

Simmel, G. (2013). El conflicto. Sociologia del antagonismo. Madrid: Sequitur.

Tavares dos Santos, J. C. (2001). A violência na escola: conflitualidade social e ações civilizatórias. Educação e Pesquisa, 27(1), 105-122. http://dx.doi.org/10.1590/S1517-97022001000100008

Tavares dos Santos, J.C. (2002). A violência na escola, uma questão social global. En R. Briceño León (Ed.). Violencia, sociedad y justicia en América Latina. (pp. 117-133.) Buenos Aires: CLACSO.

Viscardi, N. (2003). Enfrentando la violencia en las escuelas: Un informe de Uruguay. En M. Abramovay (Coord.) Violencia na escola: América Latina e Caribe. (pp. 153-205). Brasília: UNESCO.

Viscardi, N. y Alonso, N. (2013). Gramática (s) de la convivencia: un examen a la cotidianidad escolar y la cultura política en la educación primaria y media en Uruguay. Montevideo: Administración Nacional de Educación Pública.

Wieviorka, M. (2002). Le conflit contre la violence. Cosmopolitiques, (2), 12-24.

\section{Leyes}

Argentina. Ley Nº 26.892 (2013). Ley para la promoción de la convivencia y el abordaje de la conflictividad social en las instituciones educativas.

Argentina. Ley Nº 11.179 (1984). Código Penal de la Nación Argentina. 